\title{
Between reality and imagination: When is spatial updating automatic?
}

\author{
RANXIAO FRANCES WANG \\ University of Illinois at Urbana-Champaign, Champaign, Illinois
}

\begin{abstract}
Spatial updating has been shown to occur automatically and operate on both real and imagined environments. In three experiments, we compared spatial updating in real and imagined environments and examined when automatic spatial updating occurs. Participants learned the locations of real objects in a room and imagined being in their kitchens. They turned to face either the kitchen or the room targets and pointed to the targets before and after turning. Although objects in the real environment were automatically updated when the participants turned in the imagined environment, targets in the imagined environment were not automatically updated when the participants turned in the real environment. However, explicit spatial updating of imagined environments was as efficient as that of real environments. Automatic updating of the real objects required perceptual experience of these targets, either visually or by touch, and did not occur when the targets were described verbally. Implications for spatial cognition research are discussed.
\end{abstract}

Spatial updating refers to the cognitive process that allows an observer to keep track of where things are relative to herself as she changes position and orientation in an environment. Animals, including humans, constantly move from one place to another and turn from one direction to another. As an animal changes position and orientation, the spatial relationship between it and the surrounding objects changes. For example, a cup in front of someone will be to her left if she turns right. The spatial updating process keeps track of the cup's position relative to the observer as she moves, and thus allows her to grab the cup readily from the new position and orientation.

Studies have shown that spatial updating is common to many species. Animals as simple as ants can update the position of their nests relative to themselves during foraging trips by continuously adding up their movement vectors, a process referred to as path integration (see, e.g., T. S. Collett \& M. Collett, 2000; Müller \& Wehner, 1988, 1994; Wehner \& Srinivasan, 1981). Path integration is a fundamental navigation mechanism found in insects, birds, and mammals, including humans (Alyan \& McNaughton, 1999; Berthoz, Israel, Francois, Grasso, \& Tsuzuku, 1995; Etienne, Maurer, \& Séguinot, 1996; Loomis et al., 1993; Mittelstaedt \& Mittelstaedt, 1980; Müller \& Wehner, 1988, 1994; Regolin, Vallortigara, \& Zanforlin, 1995; Rieser,

This research was supported by a Research Board Grant to R.F.W. from the University of Illinois and by NSF Grant BCS 03-17681 to R.F.W. Thanks to Rashad A. Abdul-Salaam for his help in data collection and to MichelAnge Amorim, Albrecht Inhoff, Bernhard Riecke, and Heinrich Bülthoff for helpful comments on an earlier draft of the manuscript. Correspondence concerning this article should be addressed to R. F. Wang, Department of Psychology and Beckman Institute, 603 E. Daniel St., Room 533, University of Illinois, Champaign, IL 61820(e-mail: francesw@ s.psych.uiuc.edu).

Note-This article was accepted by the previous editorial team, headed by Neil Macmillan.
Ashmead, Talor, \& Youngquist, 1990; Saint Paul, 1982; Wehner \& Srinivasan, 1981). Moreover, it has been shown that spatial updating occurs automatically. For example, ants update the homing vector not only on their way out, but also during their returning journey or during detours, which suggests that the updating process is operating all the time as long as the animals move (M. Collett, T. S. Collett, \& Wehner, 1999; Schmidt, Collett, Dillier, \& Wehner, 1992). Farrell and Robertson (1998; also see Presson \& Montello, 1994, and Rieser, 1989) showed that human adults can point to target objects from a new position after they make a turn while blindfolded even if they have never seen the objects from this new perspective, which suggests that they update the targets' egocentric relationships as they turn. Furthermore, when participants were asked to ignore their movements and point to the target objects as if they had not moved, they showed significant cost in response time and accuracy, which suggests that spatial updating occurs automatically and people have great difficulty in voluntarily preventing spatial updating from occurring.

Recent studies have shown that spatial updating can operate not only on real objects but also in imagined environments. Rieser, Garing, and Young (1994) asked children to imagine being in their classrooms while they were actually sitting in the living rooms of their homes. They were asked to walk from their seats to the teacher's position as if they were in the classroom, and then to point to targets in the classroom. The actual walk greatly facilitated their performance in comparison with their simply imagining the walk, which suggests that the physical walk itself, even if it took place in the living room instead of in the classroom, allowed children to update their relationship to targets in the classroom in imagination.Loomis, Lippa, Klatzky, and Golledge (2002) asked adults to update targets either presented as 3-D sound or described verbally (e.g., "2 o'clock," "16 feet"). They showed that updating of the verbally de- 
scribed targets has the same characteristic as updating of 3 -D-sound targets, which suggests that spatial updating operates on an abstract representation that is not bound to a specific modality.

Despite the facts that spatial updating is universal across the animal kingdom, occurs automatically all the time as long as the animal has not been disoriented, and operates not only on real objects but also on imagined environments or verbally described targets, spatial updating is not immune to cognitive influences. Amorim, Glasauer, Corpinot, and Berthoz (1997) showed that spatial updating of the orientation of a target object can be influenced by participants' attentional focus. People were faster and more accurate in estimating a target object's orientation after a blindfolded walk when they paid attention to the target's orientation along the path than when they paid attention to the path itself, which suggests that the spatial updating process can be influenced by participants' attention and strategies.

These studies raised an interesting question regarding the mechanism of spatial updating. Does spatial updating occur automatically for all environments regardless of type of environment, the way the environment is learned, and number of environments? For example, when someone sits in her office, she knows where things are- the phone is to the left, the coffee mug is in front, the door is behind, and so on-even if she closes her eyes. If she then imagines herself in the middle of her kitchen facing the stove, she knows the dishwasher is to her left and the microwave is behind her. When she makes a movement relative to that imagined kitchen, however, her position and orientation relative to the real surroundings change simultaneously. What environments are included in the spatial updating process? Does she update her relationship to both the office and the imagined kitchen as she moves, or does she update only one of them?

The purpose of the present study was to address these questions. In three experiments, participants learned five target objects in a lab room (real environment) and then imagined being in their kitchens (imagined environment). They were instructed to turn to face various targets either in the real environment or in the imagined environment. Afterwards, they were tested on their knowledge of the direction of the targets in both environments. If the participants kept track of both environments as they moved, they should be able to point to targets in both environments quickly and accurately. On the other hand, if they could update only one environment at a time, they should be quick in pointing to targets in the environment in which they moved and slow in pointing to the targets from the environment in the "background."

\section{EXPERIMENT 1}

In this experiment, participants learned a set of real objects visually, were blindfolded, and imagined being in the middle of their kitchens. Then they pointed to the targets in both the real and imagined environments, before and after they turned relative to one of the environments (spatial updating). Two issues were addressed. First, is spatial updating of an imagined environment as efficient as spatial updating of real, visually learned targets? Second, does spatial updating occur automatically ${ }^{1}$ for the environment in the "background"- that is, do people update the room targets when they turn in the imagined kitchen and update their orientation in the imagined kitchen when they turn in the room?

\section{Method}

Participants. Fourteen undergraduate students from an introduction to psychology class at the University of Illinois participated for course credit.

Apparatus. The experiment was conducted in a rectangular room containing five objects (see Figure 1). The room was $8 \mathrm{ft} \times 15 \mathrm{ft}$, about the dimensions of a medium-sized kitchen in the area. An overhead video camera was mounted above the swivel chair placed in the middle of the room and recorded the entire experiment for data analysis. A Gateway 4200 PC computer randomized the target sequence for each participant.

Design and Procedure. The participants were randomly assigned to one of two groups. One group (8 participants) moved in the imagined environment (update-imagined-environment condition), and the other (6 participants) moved in the real environment (update-real-environment condition). Each participant was instructed to learn the locations of five targets in a room, to imagine being in his or her own kitchen, and to describe five targets in the kitchen. The participants were not explicitly instructed to memorize the correspondence between the two environments. However, they were instructed to sit in a swivel chair and turn around, and they were told that when they turned the kitchen would not turn with them. No explicit instructions were given about what strategies they should use, or that they would be tested about the "non-updated" environment.

Each participant was tested in three stages. In the before-updating stage, the participant was led into the lab room and sat in the swivel chair facing A (see Figure 1). ${ }^{2}$ The experimenter pointed to the five targets and the participant was allowed as much time as he or she desired to learn the targets, while free to turn in the chair. Then, the participant was blindfolded and wore wireless earphones, through which constant white noise was played and the experimental instructions and target names were delivered. Then, he or she pointed once to each target, in a random sequence, using whichever hand was most convenient. The participant was then asked to imagine being in the middle of his or her kitchen facing a familiar orientation and to name and point to five kitchen objects, starting with an object behind him or her (which later became the last target toward which he or she turned in the updating stage). This initial pointing direction was taken as the actual correct direction of the targets in the imagined environment. The participant was instructed to choose typical kitchen objects, such as refrigerator, microwave, stove, sink, dishwasher, and pantry, to distribute the targets in all four directions, and that no two targets should occupy the same horizontal direction. ${ }^{3}$ Then the participant was asked to point to the kitchen targets once each in a random order. The pointing responses made before spatial updating occurred provided a baseline measure of the participant's memory of the two environments.

In the second, updating stage, the participants were asked to turn in the swivel chair to face the five target objects in the corresponding environment in a partially random sequence while blindfolded. For the update-imagined-environment group, the participant turned to face each of the targets once in the imagined kitchen. They turned to face target after target without returning to the original orientation. For the update-real-environment group, they turned to face each of the targets in the lab room. The last target toward which they turned to face was always the poster in the update-real-environment condition and the object behind them (see the procedure for the beforeupdating stage) in the update-imagined-en vironment condition. Thus, the participants' final facing orientation in the updating stage was to- 


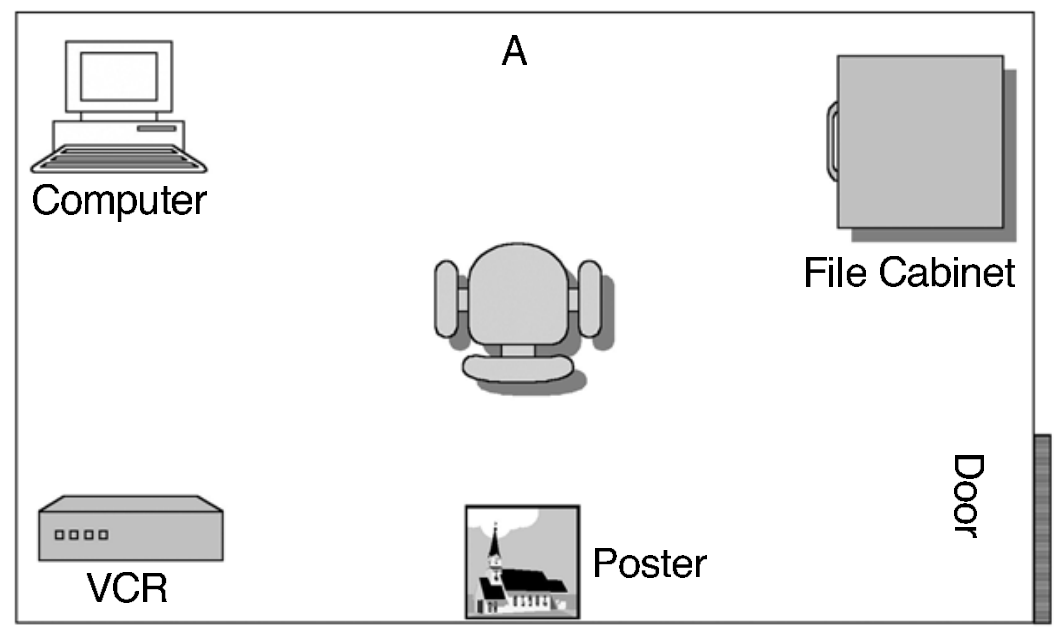

Figure 1. An overview of the test room with five targets (computer, cabinet, door, poster, and VCR) surrounding a swivel chair fixed in the middle of the room. Nontarget objects are not shown. An overhead video camera was mounted directly above the chair and photographed the overhead image of the room, which was recorded by the VCR. The participant sat in the chair and pointed to the targets while blindfolded.

ward the poster for the update-real-environment condition and toward the kitchen target closest to that direction in the update-imaginedenvironment condition.

Finally, in the after-updating stage, the participants pointed to each target once in both environments while remaining in the final position of the updating stage. Those in the update-real-environment group pointed to the targets in the lab room first, followed by the five targets in the imagined kitchen. Those in the update-imaginedenvironment group pointed to the targets in the imagined kitchen first, followed by the five room targets.

Data analysis. Both reaction time (RT) and angular pointing errors were measured on the basis of the videotape after the experiment was completed. The RT was measured from the end of the naming of the target to the completion of the response, when the participant's hand (in the case of the pointing responses) or body (in the case of the turning responses) stabilized. The direction of the pointing responses was measured by superimposing a transparent radial grid on the TV monitor centered on the participant's body, and the direction of the fingertip was taken as the pointing direction. The facing direction (for the updating stage) was measured as the direction perpendicular to the center line of the shoulders. The (unsigned) angular error was then calculated according to the actual direction of the targets.

\section{Results and Discussion}

Two questions were addressed in this experiment. First, do people update an imagined environment as efficiently as they do a real environment when explicitly required to do so? To address this issue, two measures were analyzed. Neither RT $[t(12)=1.7, p=.11]$ nor error rates $[t(12)=$ $0.82, p=.42]$ showed a significant difference between the update-real-environmentand update-imagined-environment conditions during the updating stage (for the spatial updating performance during the updating stage in all three experiments, see Table 1). Moreover, 2 (stage: before updating vs. after updating) $\times 2$ (condition: targets in the imagined environment in the update-imagined-environment condition vs. targets in the real environment in the update-realenvironment condition) analyses of variance (ANOVAs) on RT and angular errors in the pointing responses showed a main effect of stage in RT $[F(1,12)=7.1, p=.02]$, but no other effects (all $F \mathrm{~s}<2.3$, all $p \mathrm{~s}>.16$ ). Thus, both measures suggested that explicit spatial updating of an imagined environment is as effective as that of a real environment.

Second, is spatial updating automatic for an imagined environment and for a real environment? To address this issue, 2 (stage: before updating vs. after updating) $\times 2$ (target environment: imagined vs. real) ANOVAs on RT and angular errors of the pointing responses were performed for the two updating conditions. In the update-imaginedenvironment condition, there were main effects of stage

Table 1

Mean Reaction Time (RT, in Seconds) and Angular Error (Degrees) During the Updating Condition, With Standard Errors

\begin{tabular}{|c|c|c|c|c|c|c|c|c|c|c|c|c|}
\hline \multirow[b]{3}{*}{ Stage } & \multicolumn{4}{|c|}{ Experiment 1} & \multicolumn{4}{|c|}{ Experiment 2} & \multicolumn{4}{|c|}{ Experiment 3} \\
\hline & \multicolumn{2}{|c|}{ RT } & \multicolumn{2}{|c|}{$\begin{array}{c}\text { Angular } \\
\text { Error }\end{array}$} & \multicolumn{2}{|c|}{ RT } & \multicolumn{2}{|c|}{$\begin{array}{l}\text { Angular } \\
\text { Error }\end{array}$} & \multicolumn{2}{|c|}{ RT } & \multicolumn{2}{|c|}{$\begin{array}{c}\text { Angular } \\
\text { Error }\end{array}$} \\
\hline & $M$ & $S E$ & $M$ & $S E$ & $M$ & $S E$ & $M$ & $S E$ & $M$ & $S E$ & $M$ & $S E$ \\
\hline Update-imagine & 3.54 & 0.44 & 16.15 & 2.45 & 3.39 & 0.29 & 21.32 & 26.42 & 3.37 & 0.32 & 18.27 & 3.39 \\
\hline Update-real & 2.61 & 0.15 & 19.13 & 2.64 & 3.15 & 0.15 & 2.91 & 4.44 & 3.16 & 0.21 & 23.44 & 4.12 \\
\hline
\end{tabular}



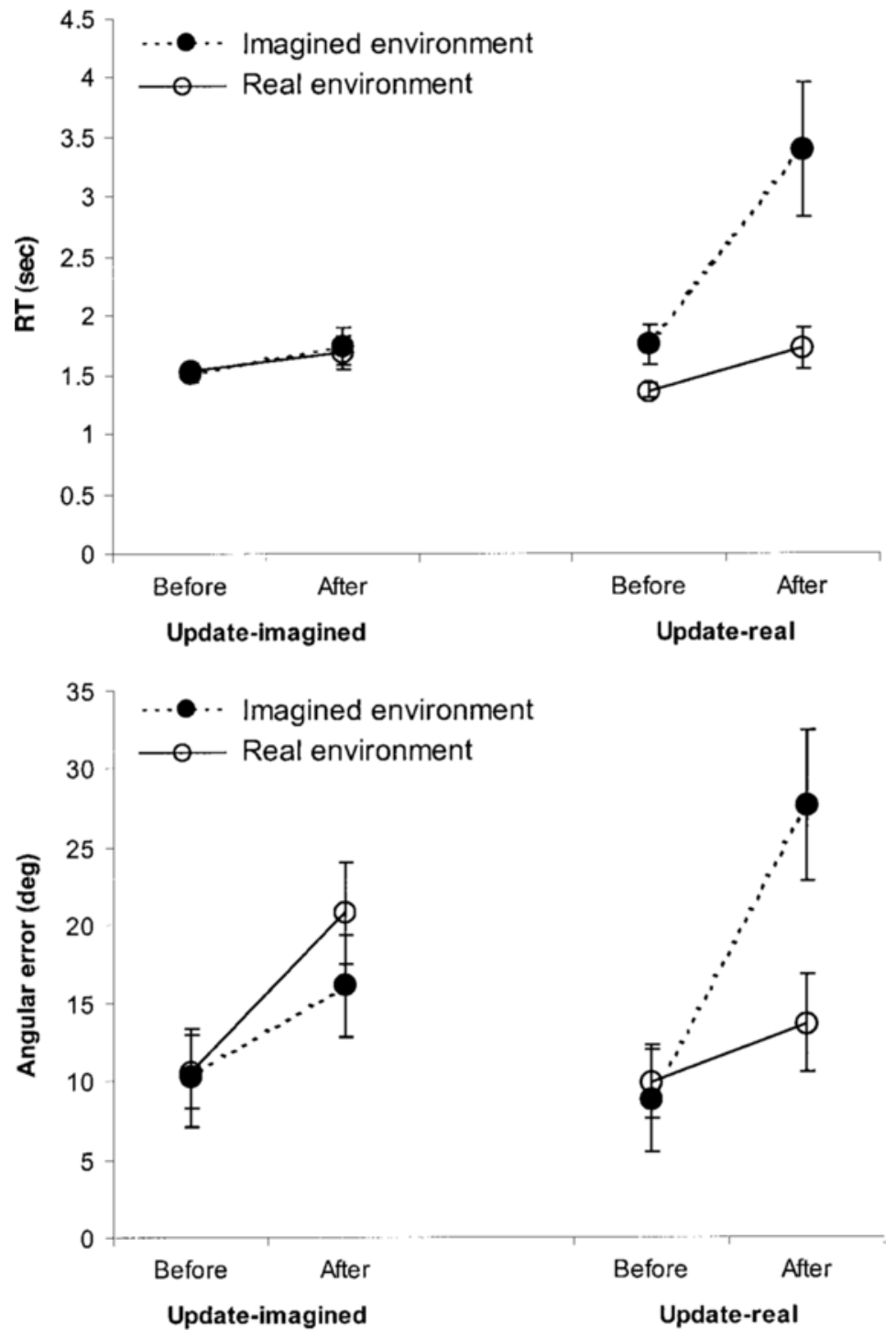

Figure 2. Reaction time (RT) and angular errors of Experiment 1 as a function of stage (before and after updating), updating condition (update-imagined and update-real), and target environment (imagined environment and real environment). The error bars represent standard errors.

[marginal for RT, $F(1,14)=4.1, p=.06$; significant for error, $F(1,14)=9.9, p=.007$ ] but no effects of target environment and no interaction between target environment and stage (all $F \mathrm{~s}<1$, all $p \mathrm{~s}>.40$ ). These results suggest that when the participants turned in the imagined environment, they updated both environments equally well (see Figure 2). In the update-real-environment condition, however, there were main effects of stage [for RT, $F(1,10)=19.8, p=.001$; for error, $F(1,10)=14.8, p=.003]$, main effects of environment [significant for RT, $F(1,10)=7.9, p=.02$; nonsignificant for error, $F(1,10)=2.7, p=.13$ ], and interactions between stage and environment [for RT, $F(1,10)=8.1$, $p=.02$; for error, $F(1,10)=6.3, p=.03]$, suggesting that the participantsupdated the real environmenteffectively but the imagined environment poorly.
To examine the effect of target stability, targets in both environments were coded as stable (large or built-in appliances such as refrigerator, stove, dishwasher, and door) or mobile (small appliances such as VCR, TV, microwave, coffee maker, and trash can). No effect of stability or mobility was found. In 2 (target stability: stable vs. mobile) $\times 2$ (stage) $\times 2$ (target environment) $\times 2$ (updating condition) ANOVAs on RT and angular errors of the pointing responses, no main effects of stability $\left(F_{\mathrm{s}}<1, p \mathrm{~s}>.42\right)$ and no interaction between stability and other factors were found $(F \mathrm{~s}<1.3, p \mathrm{~s}>.26)$. Thus, there was no evidence that target stability affected updating performance.

These results suggest that spatial updating does not always occur automatically for all environments. When the participants were instructed to turn relative to an imagined 
environment, they automatically updated their orientation relative to the real environment, showing little difference in their pointing responses to targets in both environments after the updating. On the contrary, when they were instructed to turn relative to the real environment, they effectively updated their orientation relative to the real environment but not relative to the imagined environment, showing much poorer performance in their pointing responses to targets in the imagined environment after the updating than to targets in the real environment. Thus, spatial updating is automatic for actual surroundings but not for imagined environments.

In summary, spatial updating of an imagined environment is no more difficult than spatial updating of a real environment. However, spatial updating of an imagined environment occurs only when people are required to do it. In contrast, spatial updating of the real environment occurs automatically even when people are not explicitly instructed to keep track of their orientation in the real environment.

\section{EXPERIMENT 2}

Experiment 2 was conducted to examine whether automatic updating of a real environment requires direct perceptual experience of the target objects. Participants were blindfolded, and the experimenter described the room targets to them verbally. The participants were then tested as in Experiment 1. If perceptual experience of the target objects is not necessary, then automatic updating of the real environment should occur as in Experiment 1.

\section{Method}

Twenty-three participants from the same population as in Experiment 1 were randomly assigned to the update-real-environment condition (10 participants) or to the update-imagined-environment condition (13 participants). None of them had participated in Experiment 1 . The participants were blindfolded and wore wireless earphones before they entered the testing room. Through the earphones, constant white noise was played to mask the sounds from the targets (e.g., the computer and the VCR), and experimental instructions and target names were delivered. After each participant was led to the swivel chair and sat down, the experimenter told him or her that there were five targets in the room that he or she needed to remember. Then, the experimenter named the five targets one by one and moved the participant's hand to point in the direction of that target. After the participant had learned all five targets, he or she was asked to point to the targets one by one, and any errors in the pointing responses were corrected. This procedure continued until the participant could point to all five targets within 10 degrees of their correct directions. Correction during this learning stage occurred only occasionally, and no participant required correction to a given target more than once. After this learning procedure, the participants were tested according to exactly the same procedure as in Experiment 1.

\section{Results and Discussion}

Again, the participants showed equivalent performance in their turning responses in the update-real-environment and update-imagined-environment conditions during the updating stage, both in terms of RT $[t(21)=0.7, p=.51]$ and errors $[t(21)=1.0, p=.33]$. Moreover, 2 (stage: be- fore updating vs. after updating) $\times 2$ (condition: targets in the imagined environment in the update-imaginedenvironment condition vs. targets in the real environment in the update-real-environment condition) ANOVAs on $\mathrm{RT}$ and angular errors in the pointing responses also showed no significant effects of any kind (all $F \mathrm{~s}<2.3$, all $p \mathrm{~s}>$ .14 ), suggesting that explicit updating is equally efficient for both environments.

However, unlike in Experiment 1, neither environment was automatically updated (see Figure 3). In 2 (stage: before updating vs. after updating) $\times 2$ (target environment: imagined vs. real) ANOVAs on RT and angular errors, similar patterns of results were shown in the update-realenvironment and update-imagined-environmentconditions. For the update-real-environment condition, there were main effects of stage [nonsignificant for RT, $F(1,18)=$ $2.2, p=.15$; significant for error, $F(1,18)=8.2, p=$ $.01]$, no main effects of target environment (both $F_{\mathrm{S}}<1.7$, both $p \mathrm{~s}>.21$ ), and significant interactions between stage and environment [for RT, $F(1,18)=8.8, p=.008$; for error, $F(1,18)=5.1, p=.037]$. Similarly, for the updateimagined-environment condition, there were main effects of stage [for RT, $F(1,24)=6.1, p=.02$; for error, $F(1,24)=$ $8.7, p=.007$ ], main effects of environment [nonsignificant for RT, $F(1,24)=1.4, p=.24$; significant for error, $F(1,24)=8.4, p=.008]$, and significant interactions between stage and environment [for RT, $F(1,24)=6.6, p=$ .02 ; for error, $F(1,24)=4.4, p=.05]$. These results suggest that when no direct perceptual information of the real objects is provided, spatial updating does not occur automatically for either the real environment or the imagined environment.

\section{EXPERIMENT 3}

Experiment 3 entailed a further examination of whether visual experience of the real environment is necessary for spatial updating to occur automatically. Participants were blindfolded and touched the targets before they were tested. If visual experience is not necessary, then touching the targets should allow spatial updating to occur automatically.

\section{Method}

Eighteen participants from the same population as in the previous experiments were tested in the update-imagined-environment and update-real-environment conditions, with 9 participants in each condition. None of them had participated in the previous experiments. The participants were blindfolded and sound-masked before they entered the room. After they entered the room, they were led to each of the five targets one by one in a clockwise sequence, starting at the door and traveling from target to target, and touched them. Then they were led from the file cabinet to the middle of the room and sat in the swivel chair. After that, they learned the target directions and were tested exactly as in Experiment 2.

\section{Results and Discussion}

As in Experiments 1 and 2, explicit spatial updating of the real environment was similar in efficiency to that of the imagined environment. There was no significant difference in turning performance during the updating stage 

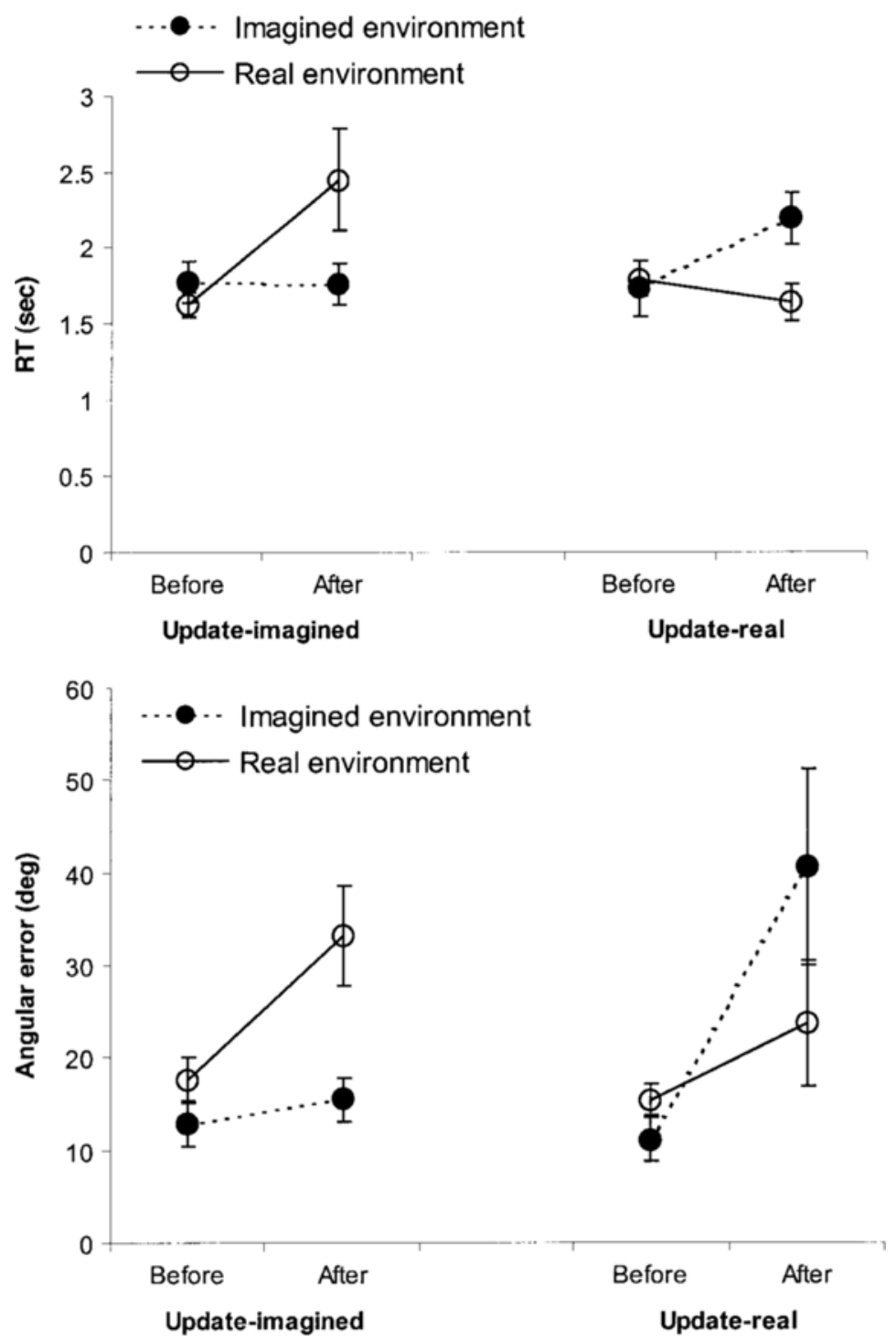

Figure 3. Reaction time (RT) and angular errors of Experiment 2 as a function of stage (before and after updating), updating condition (update-imagined and update-real), and target environment (imagined environment and real environment). The error bars represent standard errors.

[for RT, $t(16)=0.5, p=.61$; for error, $t(16)=0.9, p=$ .35]. Moreover, 2 (stage: before updating vs. after updating) $\times 2$ (condition: targets in the imagined environment in the update-imagined-environment condition vs. targets in the real environment in the update-real-environment condition) ANOVAs on RT and angular errors in the pointing responses also showed no effect on RT (all $F \mathrm{~s}<1$, all $\left.p_{\mathrm{s}}>.4\right)$. There was no effect of condition in errors $[F(1,16)=0.7, p=.42]$, although there was an effect of stage $[F(1,16)=4.6, p=.05]$ and an interaction between stage and condition $[F(1,16)=5.8, p=.03]$.

The question of interest was whether perceptual experience of the targets (through touch) allowed the participants to update the room targets automatically. The data suggest that it did, and the results paralleled those of Experiment 1 . In 2 (stage: before updating vs. after updating) $\times 2$ (target environment: imagined vs. real) ANOVAs on RT and angular errors, it was shown that in the updatereal-environment condition there were main effects of stage [nonsignificant for RT, $F(1,16)=2.2, p=.16$; significant for error, $F(1,16)=4.2, p=.05$ ], no main effects of target environment (both $F_{\mathrm{s}}<1$, both $p \mathrm{~s}>.3$ ), and interactions between stage and target environment [marginal for RT, $F(1,16)=3.7, p=.06$; significant for error, $F(1,16)=4.7, p=.04]$, suggesting that the participants updated the real environment effectively but the imagined environment poorly (see Figure 4). These results were consistent with those of Experiments 1 and 2. In the update- 

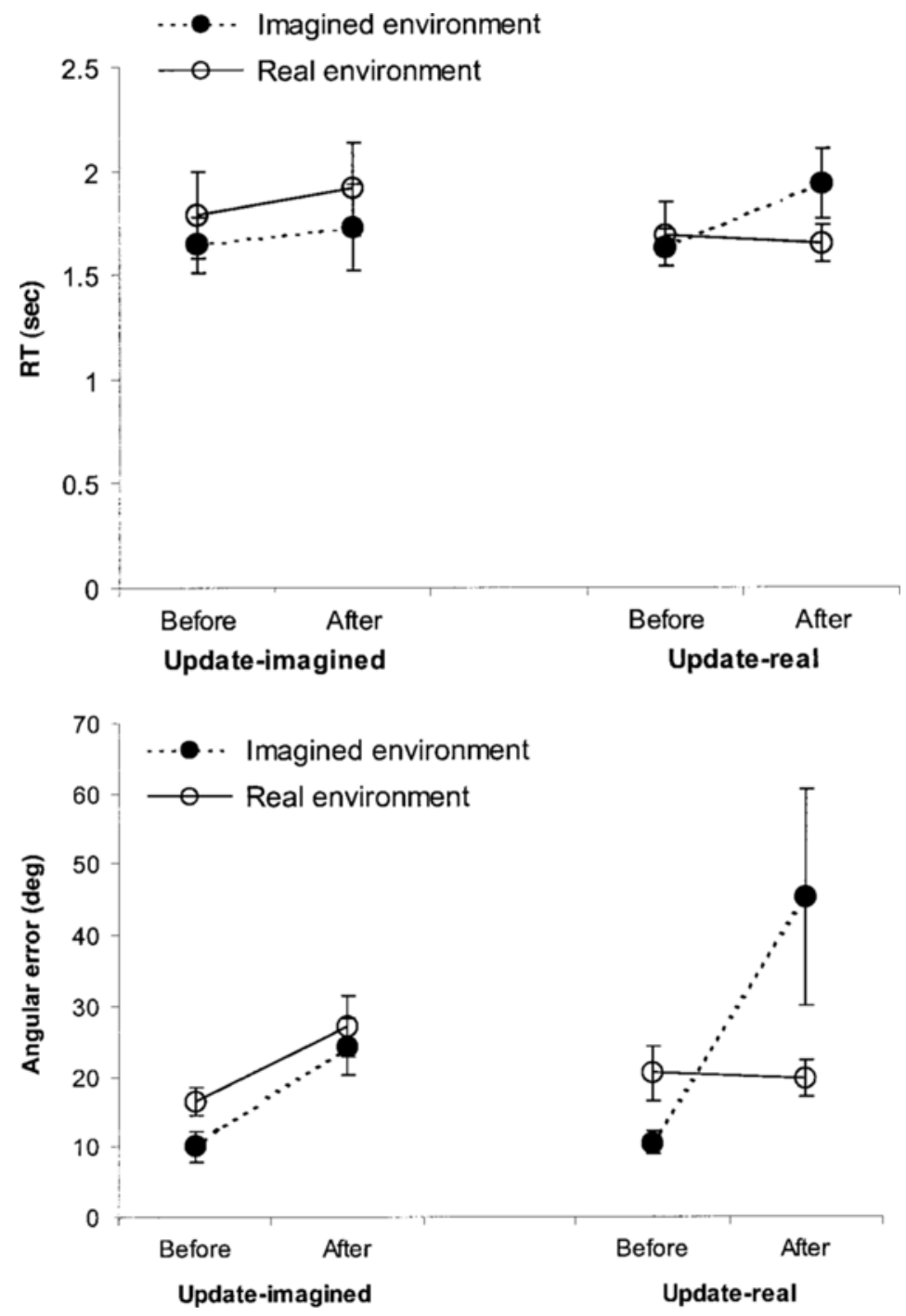

Figure 4. Reaction time (RT) and angular errors of Experiment 3 as a function of stage (before and after updating), updating condition (update-imagined and update-real), and target environment (imagined environment and real environment). The error bars represent standard errors.

imagined-environment condition, however, there was a main effect of stage for error $[F(1,16)=16.7, p<.001]$ but no other effects (all $F_{\mathrm{S}}<2.0$, all $p_{\mathrm{S}}>.17$ ). These results suggest that when the participants turned in the imagined environment, they updated both environments equally well. Thus, nonvisual experience with the real targets is sufficient to trigger automatic updating of those targets.

\section{GENERAL DISCUSSION}

In three experiments, participants learned five target objects in a lab room while they imagined being in their kitchens. Then they pointed to targets in both environments while blindfolded, before and after they turned to face objects either in the real environment or in the imagined environment. Explicit spatial updating of the imagined environment was as efficient as that of the real environment, as was shown by equivalent turning performance and the pointing responses after the updating. However, spatial updating does not always occur automatically. After the participants turned relative to the imagined environment, they showed equal performance in pointing to targets in both environments, which suggests that they automatically updated their orientation relative to the real environment. In contrast, after the participants turned relative to the real environment, they showed significantly poorer performance in pointing to targets in the imagined environment than to those in the real environment, which suggests that they failed to simultaneously update their orientation relative to the imagined environment as effectively 
as they updated their orientation relative to the real environment. Moreover, automatic updating of real environments requires perceptual experience of the target objects, and either visual or haptic information is sufficient.

These findings confirmed evidence from previous research that spatial updating of real objects in the immediate surroundings perceived visually is automatic (Farrell $\&$ Robertson, 1998) and that spatial updating can occur accurately and efficiently for imagined environments (see, e.g., Rieser et al., 1994). Moreover, these results are also consistent with previous findings that attention plays a role in spatial updating. Amorim et al. (1997) showed that the spatial updating process differed when participants attended to their own motion as opposed to when they attended to the targets. However, little is known about the updating mechanism when multiple targets or environments are involved. Here, we showed that attention may play a role in spatial updating of multiple environments. That is, two environments may sometimes, but not always, be updated simultaneously even when both environments occupy approximately the same physical space. The mechanism of spatial updating depends on the nature of the environment and on the way in which the environment is learned.

The failure to update the imagined kitchen automatically is not due to the difficulty of updating an imagined environment per se. When explicitly instructed to turn in the kitchen, the participants had no more difficulty in pointing to the kitchen targets after turning than in pointing to room targets after turning relative to the room. The failure of automatic updating of an imagined environment is also not a result of familiarity. In fact, the room was novel to the participants, who typically learned the targets in only a few minutes, whereas the kitchen was familiar to them. Moreover, the participants all had sufficient perceptual experience with their kitchens, and the targets were learned both visually and haptically through repeated experience. Thus, perceptual experience alone cannot explain the difference between spatial updating mechanisms of real and imagined environments.

One possibility is that real and imagined environments differ in their ecological consequences. Although one can imagine being in one's kitchen facing a particular orientation and knows that a stove would be in front, one also knows that if one steps forward one will not bump into a stove. However, if a real closet is in front, stepping forward will have actual behavioral consequences. Thus, updating of imagined environments is not necessary for successful locomotion. In contrast, failure to update one's spatial relationship to real objects in the immediate surroundings would constantly cause behavioral failures, and so automatic updating of the immediate surroundings is clearly a favorable trait.

These findings suggest that spatial updating relative to multiple reference frames can occur independently for each, even when the spatial relationship between these reference frames is known before the updating occurs. Wang and Brockmole (2003) showed that spatial updating relative to immediate surroundings does not automatically lead to updating relative to more remote targets. The present results showed that two environments of approximately the same scale, occupying approximately the same physical space, are not always updated simultaneously. Thus, the content of spatial updating seems to be limited.

The importance of perceptual information for the automatic updating of real objects also provides some insights into the mechanism of the spatial updating system. It appears that an object is not considered by the spatial updating system to be a real entity that can have behavioral consequences unless some assurance from the perceptual system is obtained, either visually or haptically. Although verbally described environments can be learned effectively and can be updated when one is explicitly instructed to do so, they are not treated as "real" by the spatial updating system and are not updated automatically, at least when multiple environments are present. If we consider perceptual experience as "primary learning" and verbally described or imagined relationships as "secondary learning," then spatial updating occurs for environmental representations acquired through primary learning but not for those acquired through secondary learning (Presson \& Hazelrigg, 1984; Presson \& Montello, 1994).

These findings reflect those of spatial cognition research involving moving observers. Many studies of spatial representations and navigation used fictitious environments (e.g., Franklin \& Tversky, 1990; Franklin, Tversky, \& Coon, 1992; Taylor \& Tversky, 1992, 1996), and more and more studies are using virtual reality techniques to investigate spatial cognition (e.g., Christou \& Bülthoff, 1999; Decety \& Jeannerod, 1995; Larish \& Andersen, 1995; Mallot \& Gillner, 2000; May, Peruch, \& Savoyant, 1995; Peruch, Vercher, \& Gauthier, 1995; Proffitt, Bhalla, Gossweiler, \& Midgett, 1995). However, the present findings suggest that the mechanism of spatial updating depends on the nature of the environment. That is, some of the spatial processes (such as automatic spatial updating) do not operate on verbally described or imagined environments. They also raise interesting questions about virtual environments, which are perceptually "real" even though the observer knows they are unreal. Thus, spatial updating may or may not operate automatically when people navigate in a virtual environment, and one needs to be cautious when generalizing findings obtained in virtual and imagined environments to spatial behavior in the real world.

\section{REFERENCES}

Alyan, S., \& MCNAughton, B. L. (1999). Hippocampectomized rats are capable of homing by path integration. Behavioral Neuroscience, 113, 19-31.

Amorim, M.-A., Glasauer, S., Corpinot, K., \& Berthoz, A. (1997). Updating an object's orientation and location during nonvisual navigation: A comparison between two processing modes. Perception \& Psychophysics, 59, 404-418.

Berthoz, A., Israel, I., Francois, P. G., Grasso, R., \& Tsuzuku, T. (1995). Spatial memory of body linear displacement: What is being stored? Science, 269, 95-98.

Christou, C. G., \& BülthofF, H. H. (1999). View dependence in scene recognition after active learning. Memory \& Cognition, 27, 996-1007.

Collett, M., Collett, T. S., \& Wehner, R. (1999). Calibration of vector navigation in desert ants. Current Biology, 9, 1031-1034. 
Collett, T. S., \& Colle TT, M. (2000). Path integration in insects. Current Opinion in Neurobiology, 10, 757-762.

DeCETY, J., \& JeANNEROD, M. (1995). Mentally simulated movements in virtual reality: Does Fitts's law hold in motor imagery? Behavioural Brain Research, 72, 127-134.

Etienne, A. S., Maurer, R., \& SÉGuinot, V. (1996). Path integration in mammals and its interaction with visual landmarks. Journal of Experimental Biology, 199, 201-209.

FARRELL, M. J., \& ROBERTSON, I. H. (1998). Mental rotation and the automatic updating of body-centered spatial relationships. Journal of Experimental Psychology: Learning, Memory, \& Cognition, 24, 227-233.

FRANKLIN, N., \& TVERSKY, B. (1990). Searching imagined environments. Journal of Experimental Psychology: General, 119, 63-76.

FrANKLIN, N., TVERSKY, B., \& COON, V. (1992). Switching points of view in spatial mental models. Memory \& Cognition, 20, 507-518.

LARISH, J. F., \& ANDERSEN, G. J. (1995). Active control in interrupted dynamic spatial orientation: The detection of orientation change. Perception \& Psychophysics, 57, 533-545.

Loomis, J. M., Klatzky, R. L., Golledge, R. G., Cicinelli, J. G., PelLEGRINO, J. W., \& FRY, P. A. (1993). Nonvisual navigation by blind and sighted: Assessment of path integration ability. Journal of Experimental Psychology: General, 122, 73-91.

LoOMis, J. M., LiPPA, Y., KlATZKY, R. L., \& Golledge, R. G. (2002). Spatial updating of locations specified by 3-D sound and spatial language. Journal of Experimental Psychology: Learning, Memory, \& Cognition, 28, 335-345.

MALlot, H. A., \& GILlNER, S. (2000). Route navigating without place recognition: What is recognised in recognition-triggered responses? Perception, 29, 43-55.

May, M., Peruch, P., \& Savoyant, A. (1995). Navigating in a virtual environment with map-acquired knowledge: Encoding and alignment effects. Ecological Psychology, 7, 21-36.

Mittelstaedt,M. L., \& Mittelstaedt,H. (1980). Homing by path integration in a mammal. Naturwissenschaften, 67, 566-567.

Müller, M., \& WeHner, R. (1988). Path integration in desert ants, Cataglyphis fortis. Proceedings of the National Academy of Sciences, 85, 5287-5290.

Müller, M., \& Wehner, R. (1994). The hidden spiral: Systematic search and path integration in desert ants, Cataglyphis fortis. Journal of Comparative Physiology A, 175, 525-530.

Peruch, P., Vercher, J. L., \& GaUthier, G. M. (1995). Acquisition of spatial knowledge through visual exploration of simulated environments. Ecological Psychology, 7, 1-20.

Presson, C. C., \& HazelrigG, M. D. (1984). Building spatial representations through primary and secondary learning. Journal of Experimental Psychology: Learning, Memory, \& Cognition, 10, 716722.

Presson, C. C., \& Montello, D. R. (1994). Updating after rotational and translational body movements: Coordinate structure of perspective space. Perception, 23, 1447-1455.

Proffitt, D. R., Bhalla, M., Gossweiler, R., \& Midgett, J. (1995). Perceiving geographical slant. Psychonomic Bulletin \& Review, 2, 409-428.
Regolin, L., VAllortigara, G., \& Zanforlin, M. (1995). Detour behaviour in the domestic chick: Searching for a disappearing prey or a disappearing social partner. Animal Behaviour, 50, 203-211.

RIESER, J. J. (1989). Access to knowledge of spatial structure at novel points of observation. Journal of Experimental Psychology: Learning, Memory, \& Cognition, 15, 1157-1165.

Rieser, J. J., Ashmead, D. H., TAlor, C. R., \& YoungQuist, G. A. (1990). Visual perception and the guidance of locomotion without vision to previously seen targets. Perception, 19, 675-689.

RiESER, J. J., GARING, A. E., \& Young, M. F. (1994). Imagery, action, and young children's spatial orientation: It's not being there that counts, it's what one has in mind. Child Development, 65, 1262-1278.

SAINT PAUl, U. v. (1982). Do geese use path integration for walking home? In F. Papi \& H. G. Wallraff (Eds.), Avian navigation (pp. 298307). New York: Springer.

Schmidt, I., Collett, T. S., Dillier, F. X., \& Wehner, R. (1992). How desert ants cope with enforced detours on their way home. Journal of Comparative Physiology A, 171, 285-288.

SHOLL, M. J. (1987). Cognitive maps as orienting schemata. Journal of Experimental Psychology: Learning, Memory, \& Cognition, 13, 615628.

TAYloR, H. A., \& TVERSKY, B. (1992). Descriptions and depictions of environments. Memory \& Cognition, 20, 483-496.

TAYlor, H. A., \& TVERSKY, B. (1996). Perspective in spatial descriptions. Journal of Memory \& Language, 35, 371-391.

WANG, R. F., \& BROCKMOLE, J. R. (2003). Simultaneous spatial updating in nested environments. Psychonomic Bulletin \& Review, 10, 981986.

WEHNER, R., \& SRINIVASAN, M. V. (1981). Searching behavior of desert ants, genus Cataglyphis (Formicidae, Hymenoptera). Journal of Comparative Physiology, 142, 315-318.

\section{NOTES}

1. Here, a process is defined as automatic if it occurs without explicit instruction.

2. The real environment was not impoverished as depicted in Figure 1. For clarity, the figure shows only the target layout. The room was an ordinary lab room filled with nontarget objects such as chairs, tables, cart, $\mathrm{TV}$, bulletin board, books, and small office items. Although the visual richness was difficult to match exactly, both environments had relatively natural, rich visual settings.

3 . The distributions of the target locations in the two environments were not matched. Thus, the egocentric directions differed. Previous research showed that responses were faster and more accurate for targets in front of the participants than for those behind them (Sholl, 1987). We did not find this effect in these experiments when target positions were divided into the front-half and back-half fields, possibly because of the small number of targets, and their locations were not optimal for testing the body axis hypothesis.

(Manuscript received August 30, 2002; revision accepted for publication May 14, 2003.) 